\title{
Analysis of the Cognitive Style Reflected in The Book of Changes and Traditional Chinese Medicine
}

\author{
Huang Jiangang, a \\ ${ }^{1}$ Lanzhou Petrochemical Polytechnic, Lanzhou, Gansu P. R. China \\ a465819260@qq.com

\begin{abstract}
:
The theory of traditional Chinese medicine is the application of the theory of Yijing Yin Yang, Five Elements and Qi. The thinking way of traditional Chinese medicine originates from Yijing (the Book of Changes). This paper focuses on the influence of the cognitive thinking way of Yijing on the theory of traditional Chinese medicine and the development of Chinese medicine. Although traditional Chinese medicine has its independent existence space, the cognitive thinking way of "Yi Medicine" reflects in the aspects of symmetry, cognitive bias, overgeneralization, and is unable to explore why it is. At first, the development of the theory of traditional Chinese medicine should seek a breakthrough in the aspect of traditional cognitive thinking way.
\end{abstract}

Key Words: Book of Changes, Traditional Chinese Medicine, Cognitive Thinking Way

\section{易经与中医认知思维方式的分析研究}

黄建刚 ${ }^{1, a}$

${ }^{1}$ 兰州石化职业技术学院, 甘肃, 中国

a465819260@qq.com

\begin{abstract}
摘要
中医理论是易经阴阳、五行和气的理论的应用。中医的思维方式源于易经。本文重点分析易经的认知 思维方式对中医理论及对中国医学发展的影响。虽然中医本身有其独立存在的空间, 但 “易医” 的认 知思维方式体现在对称性、认知偏差、过度概括上，且无法探索因何如此。中医理论的发展首先应当 寻求突破传统的认知思维方式。
\end{abstract}

关键词: 易经, 中医, 认知思维方式

\section{1. 前言}

现代医学进入中国后，中医在存废争议中顽强地生 长。而今中医在非器质性疾病治疗、预防保健、术后康 复方面也逐渐体现优势。虽然如此, 关于中医是否属于 科学的讨论却始终存在。这就需要我们了解中医产生的 理论基础, 了解中医在诊疗疾病方面的认知思维特点。 才能对中医准确定位。本文重点通过分析易医的认知思 维方式来探讨中医的存在价值。

2. 中医与易经观点的一致性特点

\section{1. 易经与中医 “天人合一” 的宇宙观}

\subsection{1. 中国古代 “天人合一” 的天象观}

春秋时期的 “伏羲创世” 传说这样描述：远古宇宙 “蒙蒙墨墨, 盲章预闭”,一片混沌。伏羲女娲降生分立 天地，阴阳有别，万物化育为阴阳。伏羲与女娲生的四 子即四神定立四时 “天地以设，分为阴阳，阳生于阴阴 生于阳，四维乃通”。四神又在天盖上步算时间，确定了 春分、夏至、秋分、冬至 ${ }^{[1]}$ 。1987 年发现的河南榉阳西 水坡 45 号墓星图告诉我们，6500 年前，古人已有 “天 人合一”的原始宇宙观。不过直到唐朝才最终完成将人 间帝国的一切编制到星空帝国，即二十八星宿 [2]。严格 来讲，中国古代没有天文学，只有天象学。因为中国对 
天空的观察不是研究天体演化规律, 而是为人间存在的 合理性寻找神的依据。如张衡所言: “星也者, 体生于地, 精成于天” ${ }^{[3]}$ 。

\subsection{2. 易经的宇宙观}

易经的宇宙观摈弃了古代天象观中的神话色彩。采 取推理的方式描述宇宙观。《周易・系辞上》讲: “是故 《易》有太极, 是生两仪, 两仪生四象, 四象生八卦, 八卦定吉凶” $14 \mathbf{1}$ 。这里的 “太极”、“两仪”、“四象”构 成了易经的理论框架。两仪即为阴阳, 四象衍生八卦, 既用于占卜, 也应用于中国社会的一切领域。如唐人孔 颖达在《周易正义》中解释为 “太极谓天地未分之前, 元气混而为一。” ${ }^{[5]}$ 值得注意的是, 易经不是单纯停留在 宇宙观上, 而是将乾坤的概念直接适用到人间。《易 经- 说卦传》 “乾, 天也, 故称乎父; 坤, 地也, 故称乎 母” [6]。二十八星宿, 在《易经》里对应的是河图, 河 图又演化为洛书。用先天八卦表示宇宙万物的静态关系, 描述万物的本源。

\subsection{3. 中医理论中体现的宇宙观}

传统天象观和易经里都体现了天人合一的思想, 中 医也不例外。“心者, 君主之官也, 神明出焉。肺者, 相 傅之官, 治节出焉。肝者将军之官, 谋虑出焉。胆者中 正之官, 决断出焉……凡此十二宫者, 不得相失, 故主 明则下安, 以此养生则寿” ${ }^{[7]}$ 。中医用人间君主及各级 官吏的作用来描述人体内脏各个器官的功能, 实际上是 古代天象学 “天人合一” 观点的具体应用。《黄帝内经上 古天真论篇第一》“有圣人者, 处天地之和, 从八风之理, 适嗜欲于世俗之间, 无急嗔之心.......亦可以百数”。天人 合一在中医理论中被解读为顺应四时变化、无过分忧虑、 恬静快乐为本的养生之道。

《国语・周语下》中韦昭《注》: “天有六气, 谓阴 阳风雨晦明也。地有五行, 金木水火土也。以天之六气 为经, 以地之五行为纬, 而成之也” ${ }^{[8]}$ 。后世学者怀疑 《国语》系西汉刘歆伪作。但该书毕竟记载的是公元前 990 到前 453 年历史事实, 韦昭的解释是: 十天干与十 二地支相配一周, 甲出现六次, 子出现五次, 故有 “天 六甲、地五子”。到中医里 “天有六气” 演变为风、寒、 暑、湿、燥、火六种气象。这也说明, 中医 “六气”之 说来源于传统的天象观 ${ }^{[9]}$ 。中医也看到了季节变化与人 体各脏器功能的关系。在《素问 - 六节藏象论》中说: “心者……通于夏气, 肺者……通于秋气, 肾者……通 于冬气, 肝者……通于春气” ${ }^{[10]}$, 这可能是中医认可 “天 人合一” 的朴素认识。因此, 中医的基本理论是易经宇 宙观的延申, 因此传统文化常将易经和中医并称为 “易 医”。

\section{2. 易经和中医理论均来源于简单的现实观 察}

易经和中医理论同源，孙思㝸讲：不知易者，不足 为大医。明代学者张介宾认为: 医易相通理无二致。易 经和中医均来自于简单的现实观察。易经“无极生太极, 太极生两仪” 的理论, 是建立在对周围事物的直观观察 基础上。因为我们看到的所有生命物质都是从无到有, 又归于消失。问题是为什么会 “无中生有”, 又为何 “终 究归于灭亡”, 自古至今, 无人质疑, 更无人研究。易经 用 “天人合一” 的思想囊括一切疑问。“两仪生四象，四 象生八卦”。四象在方位上指东南西北, 季节上指春夏秋 冬。可以理解古人从无到有的简单常识中, 理解天地原 来是连为一体的, 后来才有天地之别, 然后区分方位和 季节。乾 (天)、坤 (地)、艮 (山)、兑 (泽)、巽 (风)、 震 (雷)、离 (火)、坎 (水) 的八卦。所代表之物不过 是日常所见的自然物。

中医也来源于直觉观察, 除了季节变化对人体的影 响之外, 还有尝百草。也是在长期的实验观察中, 发现 某一类植物对某种疾病疗效。例如中医将砒霜作为治疗 梅毒和肺结核的辅助药物, 也是早期简单的直觉观察。 但是砒霜还是制造杀虫剂和灭鼠药的原料, 即使很谨慎 地使用少量砒霜入药, 梅毒症状可能改善, 也肯定对人 体造成了伤害。而这种伤害却是长期被忽略的。小剂量 使用砒霜给人的健康造成多大危害, 中医都没有精确的 实验分析, 直觉观察的药物疗效, 往往源于偶然性。

当同一种药材对同一种疾病产生痊愈或加重病情 的两种结果, 中医是否再继续观察深入分析该药材的疗 效呢? 我们至少在药典中没有见到具有典型药物分析 的可靠数据。

\section{3. 易经和中医的认知方式均为对称性思维}

对称性思维是中国传统文化重要的思维形式。在语 言、建筑上广泛体现, 当然在易经和中医里, 也普遍存 在对称性思维方式。阴阳构成的太极图, 是典型的对称 性思维。五行学说是土为中央, 四象即东 (木) 西 (金) 北 (水) 南 (火), 以中央土为中心, 对称分布。对称思 维形式体现的是对应和平衡。

《黄帝内经・阴阳应象大论》中, “阴阳者, 天地 之道也, 万物之刚纪也, 变化之父母, 生杀之本始, 神 明之府也。治病必求于本, 故积阳为天, 积阴为地。阴 静阳躁, 阳生阴长, 阳杀阴藏, 阳化气, 阴成行”【11】。 既然天地有阴阳, 那么治病也要分阴阳。阳气聚上为天, 阴气积下为地。这既体现了传统文化中的天人合一观念, 也反映了治病与天地对称的关系。所以将阴阳观念引入 中医, 就成了五脏 (心、肺、脾、肝和肾) 属阴, 六腑 （胃、大肠、小肠、三焦、膀胱和胆）属阳。五脏之中 又分阴阳。

这种对称性思维不可能精确地确定属种关系和同 一关系。例如《内经 - 阴阳应象大论》 “天地者, 万物 之上下也; 阴阳者, 血气之男女也; 左右者, 阴阳之道 
路也; 水火者, 阴阳之征兆也。阴阳者, 万物之始也” 【12】。前面讲了天为阳, 地为阴, 天地承载万物。那么 阴阳和 “血气之男女” 是什么关系呢? 两者没有可比性。 但是具有对称性: 阳 $\rightarrow$ 阴; 天 $\rightarrow$ 地; 男 $\rightarrow$ 女。至于为什 么将不具有同一属种关系的两个事物连接在一起, 和易 经的原理是一样的: 自古如此, 不需求证。其实质目的 就是基于天人合一的理论, 无须求真。传统文化观念里 也不允许质疑。至于阴阳则有多种解释。两仪之宇宙阴 阳是什么意思, 也不必求证, 又如东方为何属木, 西方 为何属金, 这都无需提出疑问, 只要围绕中央的对称分 布即可。很多人将对称性理解为辩证思维, 这实际上是 对哲学辩证法的曲解。对称性仅仅具有美学意义, 而作 为中医思维的一种方式, 只能是牵强附会。

\section{4. 易经和中医理论中共同的认知偏差}

认知偏差是指人们根据一定的表象或虚假的信息 而对他人作出判断, 从而出现判断失误或偏差的情况。 之所以如此, 既有人类认知过程的固有局限, 也有动机 不同。讲授《易经》的学者认为, 《易经》关于宇宙从无 到有, 从混沌中来的宇宙观, 被现代物理学关于宇宙起 源于 138 亿年前一个奇点的理论证明是正确的。在这里 需要搞清楚的是, 易经理论的创立者并没有关于宇宙发 生的科学知识。他们只是基于 “所见生命有生有灭” 的 简单常识。所以大爆炸理论不可能成为证明 “无极生太 极” 观点的正确性。传统文化中关于 “混沌初开, 先天 地生” 的观点, 仅仅源于简单的可见事物的观察。易经 讲五行相生, 即木生火, 火生土, 土生金, 金生水, 水生木。 五行相克即木克土、土克水、水克火、火克金、金克木。 木生火尚能理解, 而火生土的解释 (木被焚烧后就变成 灰烬, 灰即土), 明显牵强不能附会。还有 “金生水” 的 依据是什么? “土克水”, 为什么水不能克土? 现实中, 大洪水足以使山坍塌的例子数不胜数。按照相生相克的 直观观察的常识性解释，火为何不能克木?

中医理论中也存在这种错误的认知方式。《内经 - 金 圆真言》讲 “东风生于春, 病在肝, 俞在颈项; 南风生 于夏, 病在心, 俞在胸肋, 西风生于秋, 病在肺, 俞在 肩背; 北风生于冬, 病在肾, 俞在腰股; 中央为土, 病 在脾, 俞在脊” 【13】。很明显, 春天患感冒就不是病在肝 上。当讲 “冬气者, 病在四肢” 时, 还应注意, 今年爆 发的新型冠状病毒则是 2019 年 12 月发生的, 不在四肢, 病在肺。认知偏差常见认知误区在于: 一种理论看似构 成体系, 完全是因为不允许质疑, 由创立者任意演绎。

\section{5. 易经和中医认知思维均存在过度概括性}

过度概括是不合理归因方式的一种。这种思维方式 只能导致我们认识混乱。《易经 - 说卦传》讲: “乾为天, 为君、为父。坤为地, 为母, 为布” ${ }^{[14]}$ 。而从两仪上讲,
乾为阳, 坤为阴。用乾坤解释自然现象时为天地, 解释 政治隶属关系时为君臣、解释家庭关系时为夫、妻。显 然天地、君臣和夫妻关系不属于一个属种关系。将阴阳 比作天地, 如果是一种方位概念的区别还能解释。那么 将乾（阳）坤（阴）又比做男女、父母就不是方位概念 了。过度概括源于对不同概念缺乏准确的定义, 以认知 者的偏好将毫无属种关系的概念混为一谈。易经将不具 有同一属种关系的认识对象放在一起, 显然是有目的的:

“天尊地卑，乾坤定矣。卑高以陈，贵贱位矣” [15】。将 自然关系 (天地) 分为尊卑, 目的是将此类比人间, 以 此确定人间的贵贱地位。

易经上的阴阳具有不确定性，可以任意扩大适用范 围。应用于中医理论就同样是经不起推敲的。

在中医里, “阴阳”也没有准确定义。所以 “阴阳” 概念就可以随意使用。对阴阳所确定部位进行的任何解 释都是正确的。《内经》关于阴阳的区分标准有三个:

“背为阳, 腹为阴”, “五脏属阴, 六腑属阳”、“腰以上 为阳, 腰以下为阴”。那么六腑为何属阳? 五脏为何属 阴？既然五脏在腰以上, 又如何解释五脏属阴？中医采 用易经 “阴中有阳、阳中有阴”的理论。将这种混乱的 阴阳关系随意解释, 想当然地将其 “合理化”。由于概念 没有准确的定义, 必然导致我们不能深入细致的研究, 更无法发现中医理论的不足并推动中医理论的完善与 进步。

\section{6. 易经和中医中因果联系的不必然性}

只有符合因果必然联系的联想才有利于我们认知 的进步。 $\mathrm{A}$ 事物是 $\mathrm{B}$ 事物的原因。那么在研究为什么 $\mathrm{B}$ 时, 只要发现 A 即可。在诊断疾病时, 我们就是根据症 状 (B) 来寻找出现 B 的原因 A。找到产生疾病的原因, 才能对症治疗。当试图解决问题时, 只有当联想符合因 果必然联系，才更有利于找到解决问题的方法。那么中 医理论是否如此呢?

《易经・彖传上》对泰卦的解释: “泰, “小往大来, 吉, 亨, , 则是天地交而万物通也; 上下交而其志同也” [16]。天地阴阳交合万物畅通，与君臣上下交流才能志同 道合，问题是天地交和与君臣沟通之间并没有逻辑必然 性。还有前面所讲, 乾为天、为君、为父, 坤为地、为 臣、为母中 “天、君、父” 和 “地、臣、母”一样都不 是同义语词，也不具有对等关系。那么何以用乾坤概括? 没有因果必然性的联想就是耗散的。同一件事，不同心 理感受的人, 会产生不同的联想：面对患癌病人去世, 有人感叹生命脆弱, 有人警示自己经常体检。这完全归 因于个人的情境联想。

根据艾里斯情绪的 $\mathrm{ABC}$ 理论: “认为激发事件 A（病 人死亡) 只是引发情绪和行为后果 $\mathrm{C}$ (不同人的不同联 想）的间接原因，而引起 C 的直接原因则是个体对激发 事件 $\mathrm{A}$ 的认知和评价而产生的信念 B (自己的感情体验)。 即人的消极情绪和行为障碍结果 ( $C$ 一不同人的不同感 叹), 不是由于某一激发事件 ( $\mathrm{A}$-病人死亡) 直接引发的, 而是由于经受这一事件的个体对它不正确的认知和评 
价所产生的错误信念 (B-不同人的不同联想) 所直接引 起。错误信念也称为非理性信念。

《内经・阴阳应象大论》提出: “东方青色, 生风, 风生木, 入通于肝, 藏精于肝, 其病发惊骇; 南方赤色, 入通于心。生热, 热生火, 火生苦, 苦生心。藏精于心, 病在五脏; 中央黄色, 生湿, 湿生土, 土生甘, 甘生脾, 入通于脾。藏精于脾, 病在舌本; 西方白色, 入通于肺。 藏精于肺, 故病在背。生燥, 燥生金, 金生辛, 辛生肺; 北方黑色, 入通于肾, 藏精于肾, 故病在溪。生寒, 寒 生水, 水生咸, 咸生肾” ${ }^{1} 17 \mathrm{r}$ 。那么肝主木, 心主火、脾 主土、肺主金、肾主水的依据是什么? 中医常讲 “怒伤 肝”, 但喜怒过度引发的是心血管疾病, 却是不争的事 实。

易经的认知思惟, 是将两个没有因果必然联系的事 物牵强附会, 在卜筮解卦时, 似乎存在 “合理性解释”。 但是这种思维方式的危害是巨大的。长此以往, 耗散式 的联想总是误导我们随意解释事物之间的关联关系。例 如中国天象观中 “浑天说”, “天地浑沌如鸡子。盘古生 在其中。万八千岁。天地开辟” ${ }^{\mathbf{1} 181}$ 。那么天地如鸡子的 依据是什么? 盘古在其中待了 1 万八千年的依据在哪里? 不过是随意联想而已。易经理论中普遍存在不遵循逻辑 关系的认知思维现象。如《易经・彖传下》这样解释天地 人关系: “天地感, 而万物化生; 圣人感人心, 而天下 和平” ${ }^{1191}$ 。这里万物化生与圣人感人心没有逻辑关系, 同样 “圣人感人心” 与实现天下和平没有必然联系。

\section{3. 中医的诊疗定位}

前述分析可以看出, 用易经理论武装的中医, 在理 论体系上的确存在被质疑的理由。那么中医就真的无用 吗? 显然不是, 这就需要我们寻找中医的核心价值, 准 确定位中医。

我们先看中医如何诊断肾阴虚和肾阳虚: 白天手心 出汗、体内燥热通常被诊断为肾阳虚。夜晚盗汗梦遗常 被诊断为肾阴虚。对肾阴虚患者服用六味地黄丸、知柏 地黄丸都是可以的, 平时多吃苦瓜、番茄、荠白、荣荠、 菱肉、百合等。对肾阳虚患者服用金贵肾气丸、桂附地 黄丸, 日常适当多食山药、肉苁蓉、杜仲等。如果我们 不用阴阳学说, 诊疗肾脏疾病, 夜晚盗汗梦遗、白天手 心出汗燥热各如何用服药保养, 也同样达到对症下药的 目的。这个例子是要说明, 中医是长期直观观察的产物。 只不过是用易经理论武装而已。我们再看人体穴位（腧 穴)。穴位多为神经末梢和血管较多的地方, 主要指人体 经络线上特殊的点区部位。古代医生非常了不起, 能够 在长期的直观观察中发现人体 720 个穴位。中医可以通 过针炎或者推拿、点按、艾炎刺激相应的经络点治疗疾 病。虽然我们还不知道这些穴位的本质, 但至少中医针 炎治疗偏痽、中风患者效果要超过西医。所以不存在废 除中医的问题, 而在于中医独立的生存环境里, 如何准 确把握中医问题。

\section{4. 如何发挥中医的优势}

以肾炎病历说明，患者的 “肾虚” 没有达到西医确 定的“肾炎”标准时, 西医常常是嘱咐就医者注意休息、 劳逸结合。问题是患者的临床症状, 说明患者至少存在 亚健康的状态。中医就可以做到防患于未然：可以通过 饮食调整, 结合草药调理, 就可以达到健康状态。中医 还对病人手术后恢复人体机能方面具有积极意义。

诊疗疾病时, 不知道疾病发生的原理, 就不可能对 症诊疗。现代科学研究证明, 肤腺细胞中的胰岛 $\beta$ 细胞 分泌胰岛素, 胰岛细胞功能损坏造成胰岛素分泌不足, 就是糖尿病的根本原因。但是导致胰岛功能损坏的原因 却很复杂。中医认为, 糖尿病是消渴症, 是从五行之说 解释糖尿病的。由于它不是从生物学原理解释疾病的本 质, 所以中医治愈糖尿病就没有必然性。问题在于, 造 成胰岛细胞功能损坏的原因非常复杂，例如饮食不当、 缺乏运动不能有效消耗糖分都可以诱发糖尿病。因此患 者血糖在 7.0 以内时, 西医强调加强锻炼、控制饮食, 很有可能使糖尿病患者逆转。而中医主张饭吃七分饱, 反对暴食暴饮, 主张荤素搭配及有规律的作息时间。很 显然, 这对预防糖尿病是有积极作用的。

再看人体穴位 (腧穴), 在早期预防保健方面中医优 势比较明显。易医的认知思维方式决定了中医重点倾向 于外部环境对人体的影响, 强调人要顺应自然 (如四季 变化）的规律。中医诞生以来 2000 多年的历史, 始终 贯穿 “天人合一” 的思想。准确地说, 中医是诊疗疾病 为辅、预防为主的保健养生医学。对于因细胞损坏、病 毒或基因变异有关的疾病, 西医疗效显著, 中医则显不 足。因为两者不是一个认知思路。认知思路不用, 解决 问题的手段也就不同。我们发扬中医, 不是要与西医比 高低, 而是注意中西医各自独特的优势。

\section{项目基金}

2019年甘肃省哲学社会科学规划项目《中 国传统文化与中医的 “天人合一”》课题 Chinese Traditional Culture and Oneness of Heaven and Man of Traditional Chinese Medicine Philosophy and Social Science Planning Project of Gansu Province in 2019

\section{References}

[1] Archaeoastronomy in China written by Fen Shi p60 China Academy of Social Sciences Press May 2017 Third Edition

[2] Ancient Religion and Ethics Written by Chen Lai P25 Peking University Press April 2017, First Edition

[3] Starry Empire: Secrets of Ancient Stars in China Written by Xu Gang, Wang Yanping, p5--p9 Posts and Telecom 
Press, August 2016, First Edition

[4] Yijing Pedigree Written by Song Xuehai, Wang Hui, p423 Yunnan People's Publishing House, October 2013, First Edition

[5] Yijing Pedigree Written by Song Xuehai, Wang Hui, p423 Yunnan People's Publishing House, October 2013, First Edition

[6] Yijing Pedigree Written by Song Xuehai, Wang Hui, p423 Yunnan People's Publishing House, October 2013, First Edition

[7] The Yellow Emperor's Classic of Internal Medicine. Written by Gao Jinghua, p23 Shanxi Science \& Technology Press

[8] Archaeoastronomy in China written by Fen Shi p226 China Academy of Social Sciences Press May 2017 Third Edition

[9] Archaeoastronomy in China written by Fen Shi p226 China Academy of Social Sciences Press May 2017 Third Edition

[10] The Yellow Emperor's Classic of Internal Medicine. Written by Gao Jinghua, p26 Shanxi Science \&Technology Press

[11] The Yellow Emperor's Classic of Internal Medicine. Written by Gao Jinghua, p22 Shanxi Science \&Technology Press

[12] The Yellow Emperor's Classic of Internal Medicine. Written by Gao Jinghua, p15 Shanxi Science \&Technology Press

[13] The Yellow Emperor's Classic of Internal Medicine. Written by Gao Jinghua, p09 Shanxi Science \&Technology Press

[14] Yijing Pedigree Written by Song Xuehai, Wang Hui, p456 Yunnan People's Publishing House, October 2013, First Edition

[15] Yijing Pedigree Written by Song Xuehai, Wang Hui, p406 Yunnan People's Publishing House, October 2013, First Edition

[16] Yijing Pedigree Written by Song Xuehai,Wang Hui, p318 Yunnan People's Publishing House, October 2013, First Edition

[17] The Yellow Emperor's Classic of Internal Medicine. Written by Gao Jinghua, p14 Shaanxi Science \&Technology Press

[18] External History of Tianxue Written by Jiang Xiaoyuan p117 Shanghai Jiao Tong University Press
[19] Yijing Pedigree Written by Song Xuehai, Wang Hui, p327 Yunnan People's Publishing House, October 2013, First Edition 